\title{
Magnetic field morphologies at mpc scale
}

\author{
Ya-Wen Tang ${ }^{1,2}$, Patrick M. Koch ${ }^{3}$, Paul T. P. Ho ${ }^{3,4}$, \\ Stephane Guilloteau ${ }^{1,2}$ and Anne Dutrey ${ }^{1,2}$ \\ ${ }^{1}$ LAB, France $;{ }^{2}$ CNRS, France; ${ }^{3}$ ASIAA, Taiwan; ${ }^{4}$ CfA, USA
}

We report our new results of the magnetic field (B) morphologies toward W51 North,traced with the linear polarization of the dust continuum at wavelengths of $870 \mu \mathrm{m}$. The B morphologies are resolved with an angular resolution of typically 1" using the Submillimeter Array (SMA). Dense structures with a number density $10^{5}$ to $10^{7} \mathrm{~cm}^{-3}$ are traced. In comparison, the B morphologies of sources at different evolutionary stages, from the collapsing core in W51 e2 (Tang et al. 2009a) and part of Orion BN/KL (Tang et al. 2010) to the ultra-compact HII region G5.89-0.39 (Tang et al. 2009b) clearly exhibit different morphologies, likely suggesting different roles of the B fields at different stages.

In the W51 North region we analyze field structures at three different physical scales (Tang et al. 2012). In a sequence of increasingly higher resolution observations - from $\mathrm{CSO} / \mathrm{JCMT}$ single dish at $2 \mathrm{pc}$ to the SMA highest resolution at about $10 \mathrm{mpc}$ - it becomes manifest how the field morphologies change from the envelope surface layer to the inner core and disk. Structures vary from uniform to cometary and hourglass-like. We quantify these changes, providing evidence that the interplay of the B field with other forces, such as gravity, evolves with scale. Additionally, new analysis methods to interpret these observational results and to derive B field strength maps are also discussed (Koch et al. 2012a,b,c).

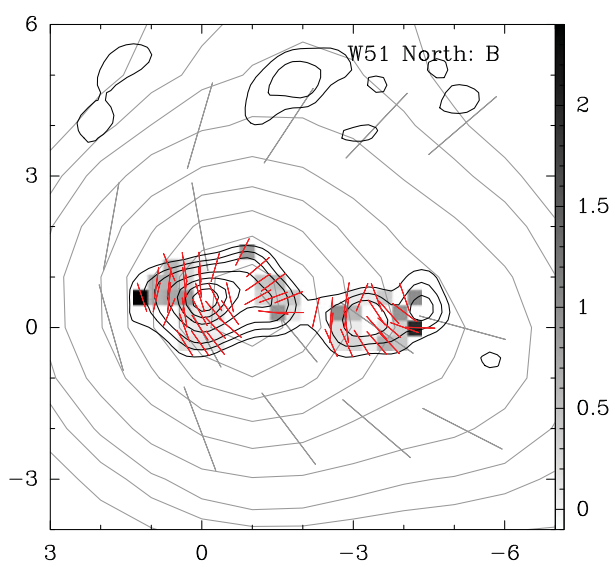

Figure 1. The figure in the left presents the $B$ field morphologies observed in two different SMA configurations, which trace the B fields at two different scales. The dust continuum emission traced at these two scales is shown in contours. The magnetic-field-togravity force ratio map, derived from our newly developed method, is shown in grey scale. The ratio is smaller toward the denser regions, suggesting a varying role of the $\mathrm{B}$ field as a function of scale.

\section{References}

Koch, P. M., Tang, Y.-W., \& Ho, P. T. P. 2012a, ApJ, 747, 79

Koch, P. M., Tang, Y.-W., \& Ho, P. T. P. 2012b, ApJ, 747, 80

Koch, P. M., Tang, Y.-W., \& Ho, P. T. P. 2012c, ApJ, submitted

Tang, Y.-W., Ho, P. T. P., Girart, J. M., et al. 2009a, ApJ, 695, 1399

Tang, Y.-W., Ho, P. T. P., Koch, P. M., et al. 2009b, ApJ, 700, 251

Tang, Y.-W., Ho, P. T. P., Koch, P. M., et al. 2010, ApJ, 717, 1262

Tang, Y.-W., Ho, P. T. P., Koch, P. M., et al. 2012, ApJ, submitted 